Article

\title{
Research and Application of Hybrid Wind-Energy Forecasting Models Based on Cuckoo Search Optimization
}

\author{
Ru Hou ${ }^{1}$, Yi Yang ${ }^{2, *}$, Qingcong Yuan ${ }^{3}$ and Yanhua Chen ${ }^{4}$ \\ 1 School of Mathematics \& Statistics, Lanzhou University, Lanzhou 730000, China; houru5101@163.com \\ 2 School of Information Science and Engineering, Lanzhou University, Lanzhou 730000, China \\ 3 Department of Statistics, Miami University, 304 B Upham Hall, Oxford, OH 45056, USA; \\ qingcong.yuan@miamioh.edu \\ 4 School of Information Engineering, Zhengzhou University, Zhengzhou 450000, China; \\ ieyanhuachen@zzu.edu.cn \\ * Correspondence: yy@lzu.edu.cn
}

Received: 6 August 2019; Accepted: 20 September 2019; Published: 26 September 2019

\begin{abstract}
Wind energy is crucial renewable and sustainable resource, which plays a major role in the energy mix in many countries around the world. Accurately forecasting the wind energy is not only important but also challenging in order to schedule the wind power generation and to ensure the security of wind-power integration. In this paper, four kinds of hybrid models based on cyclic exponential adjustment, adaptive coefficient methods and the cuckoo search algorithm are proposed to forecast the wind speed on large-scale wind farms in China. To verify the developed hybrid models' effectiveness, wind-speed data from four sites of Xinjiang Uygur Autonomous Region located in northwest China are collected and analyzed. Multiple criteria are used to quantitatively evaluate the forecasting results. Simulation results indicate that (1) the proposed four hybrid models achieve desirable forecasting accuracy and outperform traditional back-propagating neural network, autoregressive integrated moving average as well as single adaptive coefficient methods, and (2) the parameters of hybrid models optimized by artificial intelligence contribute to higher forecasting accuracy compared with predetermined parameters.
\end{abstract}

Keywords: wind speed forecasting; data pre-analysis; parameter optimization; cuckoo search algorithm

\section{Introduction}

Fossil fuel resources are not renewable and are very limited, therefore, it is necessary to seek sustainable and clean energy resources. However, in practice, one problem with utilizing wind energy is that, compared with other electricity-generating methods, wind energy is not as steady and it is intermittent. Furthermore, if the electricity from wind energy accounts for a fairly large proportion of total electricity supply, the safe operation of the power grid may be threatened. Many factors, such air density around the wind farms and wind turbine characteristics, have an impact on the wind energy production. The use of energy storage systems can eliminate short-term disconnections from the grid of wind-energy sources [1]. Specifically, the amount of energy in wind is related with the cubic of the wind speed [2]. Therefore, the accuracy of wind-speed forecasting must be improved to attain more precise estimates of wind-power generation and thus better guide power dispatching and scheduling. This will to some extent promote the wind-energy industry to generate wind power more efficiently and ensure sustainable development. 
Wind-speed forecasting methods can be divided by researchers into different fields contributing to develop models to forecast wind speed. For example, physical, statistical, or artificial intelligence models. Other models that involve multiple methods are also proposed, such as the combined models and hybrid models. The physical model utilizes wind farm and geographic information around the wind turbine to obtain the wind-speed data and other values of interest. It is usually very complex and has high precision and wide application in long-term prediction. The statistical model describes the mapping relationship between various parameters input by the system and the wind farm output, mainly including the time series method [3-5], exponential smoothing method [6], Kalman filtering method [7], regression method [8,9], empirical mode method [10-13] and so on.

Generally speaking, artificial intelligence models are the most widely used wind-speed forecasting models. For example, Blanchard and Samanta [14] proposed the non-linear autoregressive neural network method and non-linear autoregressive neural network with exogenous inputs method, which are two variations of artificial neural networks. Chitsazan et al. [15] utilizes the non-linear relationship between the internal states of echo state networks (ESN) and the proposed two methods for wind direction and speed forecasting. Compared with the classical ESN methods, the proposed method is computationally more efficient. It reduces the order of the weight matrices and has a smaller number of internal states.

A novel wind-speed forecasting method which utilizes an optimal model selection strategy is proposed by Zhou et al. [16]. The method first uses the improved boxplot to de-noise the data, then back propagation $(B P)$, wavelet neural network $(\mathrm{WNN})$, general regression neural network (GRNN) and adaptive network-based fuzzy inference system (ANFIS) are applied for the forecasting step, then finally, WIC is adopted to find the best model. Hu et al. [17] developed a hybrid model to do wind forecasting based on ensemble empirical mode decomposition (EEMD) and support vector machine (SVM), and the obtained experimental results reported an observable improvement of forecasting accuracy. Based on multi-scale dominant ingredient chaotic analysis, Fu et al. [18] present a novel hybrid method. It improved the hybrid GWO-SCA (IHGWOSCA) algorithm and extreme learning machine (ELM) for multi-step short-term wind-speed prediction. A new hybrid method which predicts the conditional quantile of wind speed was proposed by Zhang et al. [19]. This approach combines quantile regression and minimal gated memory network methods. They also discussed the feature selection and combination's goal in constructing the optimal feature inputs. Li et al. [20] proposed a new wavelet packet decomposition (WPD)-Boost Elman neural network (ENN)-wavelet packet filter (WPF) prediction method that combines WPD, ENN, boosting algorithms and WPF. The analysis show competent results of the proposed method in big multi-step wind-speed prediction.

Data pre-analysis techniques are frequently employed by researchers to improve forecast accuracy and powerful strategies. For example, Wu et al. [21] developed a deep feature extraction approach, which relied on stacked denoising autoencoders and batch normalization. Using this method, the forecasting accuracy of a long short term memory network (LSTM) is $49 \%$ more than traditional feature selection method. This indicates that it is of importance of choose a proper feature extraction method to forecast the wind speed. A Kalman filtering technique was demonstrated by Cassola and Burlando [22] to forecast wind speed and power. It was shown that this method can provide significant forecast improvement for short-term forecast. Then Kalman-filtered wind-speed data were used to forecast the wind energy output. Utilizing the Kalman-filtering method, the difference between the predicted and actual wind energy values during two years is very small. It also showed a stable evolution. The percentage error of a testing for two years between simulated and measured wind-energy values was still very low and showed a stable evolution. Niu et al. [23] employed a complete ensemble empirical mode decomposition with adaptive noise (CEEMDAN) method to de-noise the wind-speed data. The authors highlighted that the application of CEEMDAN was helpful to eliminate possible systematic errors and could get more accurate forecasting results. Chen and $\mathrm{Yu}$ [24] proposed a support vector regression-unscented Kalman filter (SVR-UKF) method to do wind speed's short-term 
estimation. They first used SVR to formulate a non-linear state-space model, then dynamic state estimation was done using the UKF method recursively.

As seen above, conventional methods such as linear regression and time series analysis are not sufficient for wind-speed forecasting. Many complex models based on modern approaches such as neural networks have been proposed and tested using real wind-speed data. Different techniques, such as a Kalman filter, wavelet packet decomposition and seasonal exponential adjustment, are used to preprocess the original data to get better forecast performance. In addition, hybrid models and comparative analysis are frequently exploited to obtain optimal forecasting results. Considering that wind speed is impacted by many factors with obvious local characteristics that are difficult to identify completely and measure precisely, the algorithms and models that provide acceptable accuracy usually vary with different data sources. Although extensive research has been conducted on wind-speed forecasting in recent years, new forecasting methods are highly needed, especially methods that are efficient and have high forecasting accuracy under specific circumstances.

The contribution of the present paper is to propose new hybrid models for predicting long-term wind speed. In particular, a total of four types of hybrid strategies based on seasonal exponential adjustment, adaptive coefficient methods and the cuckoo search algorithm are proposed. We differentiate the seasonal fluctuation and inherent trends of the original data using a seasonal exponential adjustment method. In addition, the cuckoo search algorithm is employed for parameter estimation in the adaptive coefficient method. The significance of the developed forecasting techniques is that adaptive coefficient methods are capable of adaptively capturing non-linear and non-stable patterns in the data because the endogenous parameters are calculated and adjusted simultaneously according to forecast errors. In particular, high exploitation capability of the artificial intelligence searching algorithm enables it to find much more optimal exogenous parameters of adaptive coefficient methods than predetermined ones, and thus the forecasting results can be further improved.

The rest of the paper is organized as follows. In Section 2, methodologies of the hybrid forecasting models are introduced, including the seasonal exponential adjustment technique, the adaptive coefficient method, and the cuckoo search algorithm. Section 3 presents the proposed four hybrid forecasting models. Section 4 shows case studies and forecasting results from four observation sites located in Xinjiang Uygur Autonomous Region, China. Finally, Section 5 reports the relevant conclusions to sum up this study.

\section{Related Methodology}

In this section, three components of the proposed hybrid forecasting strategies are presented, namely, the seasonal exponential adjustment, the adaptive coefficient method and the cuckoo search algorithm, which are applied to data preprocessing, trend forecasting and parameter optimization, respectively.

\subsection{Seasonal Exponential Adjustment}

Because periodic components and trend items usually coexist in long-term wind-speed data, seasonal exponential adjustment is carried out to calculate seasonal indices and to separate the inherent trend patterns from the original dataset. The procedure of this data preprocessing technique is illustrated in detail below. Note that there are generally two kinds of assumptions about the relationship between periodic and trend components: multiplication and addition [25].

First, with respect to seasonal exponential adjustment in the multiplicative form, suppose $y_{t}$ is used to represent the wind speed at time $t$, and

$$
y_{t}=S_{t} * R_{t}
$$


where $R_{t}$ represents the trend item and $S_{t}$ is the cycle item. Rearrange the wind-speed time series $y_{1}, y_{2}, \cdots, y_{T}$ to

$$
y_{11}, y_{12}, \cdots, y_{1 l} ; \cdots ; y_{k 1}, y_{k 2}, \cdots, y_{k l} ; \cdots ; y_{m 1}, y_{m 2}, \cdots, y_{m l}(T=m * l)
$$

which means that there are $l$ data items in each cycle and $m$ cycles in the dataset. The average of $y_{t}$ in each cycle is usually used to substitute the unknown trend component, which can be calculated as

$$
\bar{y}_{k}=\frac{1}{l} \sum_{s=1}^{l} y_{k s}, k=1,2, \cdots, m
$$

Suppose

$$
S_{k j}=\frac{y_{k j}}{\bar{y}_{k}}, k=1,2, \cdots, m ; j=1,2, \cdots, l
$$

and then the seasonal index can be computed as follow:

$$
I_{j}=\frac{S_{1 j}+S_{2 j}+\cdots+S_{m j}}{m}, j=1,2, \cdots, l
$$

Divide the original wind-speed data by the calculated seasonal indices in turn as follows:

$$
y_{k j}^{\prime}=\frac{y_{k j}}{I_{j}}, k=1,2, \cdots, m ; j=1,2, \cdots, l
$$

Then, the preprocessed time series without the effect of seasonal factors $y_{11}^{\prime}, y_{12}^{\prime}, \cdots, y_{1 l^{\prime}}^{\prime} ; \cdots ; y_{k 1}^{\prime}, y_{k 2}^{\prime}, \cdots, y_{k l}^{\prime} ; \cdots ; y_{m 1}^{\prime}, y_{m 2}^{\prime}, \cdots, y_{m l}^{\prime}$ can be obtained. Obviously, the series can be rewritten as $y_{1}^{\prime}, y_{2}^{\prime}, \cdots, y_{T}^{\prime}$, which indicates the targeted inherent trend components of the original data.

Second, when considering seasonal exponential adjustment in the additive form, we assume the wind speed at time $t$ can be represented as

$$
y_{t}=R_{t}+S_{t}
$$

where $R_{t}$ refers to the trend item and $S_{t}$ is the cycle item. Similarly, the original wind-speed time series can be rearranged as described above, and the average value of each cycle can be calculated according to Equation (2). However, under the assumption of addition, suppose

$$
S_{k j}=y_{k j}-\bar{y}_{k}, k=1,2, \cdots, m ; j=1,2, \cdots, l
$$

Then the seasonal index can be computed as shown in Equation (4). Subtract the calculated seasonal indices from the original wind-speed data as follows:

$$
y_{k j}^{\prime}=y_{k j}-I_{j}, k=1,2, \cdots, m ; j=1,2, \cdots, l
$$

Then, the new time series without seasonal fluctuations are obtained for further forecast work.

In summary, the seasonal exponential adjustment technique, in multiplicative or additive form, is performed directly on the original dataset to get rid of seasonal components, which are represented as calculated seasonal indices. Furthermore, only the trend items are targeted for forecasting model construction. Once the trend items are forecasted, the corresponding seasonal indices will be multiplied or added back to get the final forecasting results. For example, suppose the forecasted trend value at time $T+1$ is $y_{T+1}^{\prime}$, and then the final forecast value $\hat{y}_{T+1}$ should be calculated as in the multiplicative form

$$
\hat{y}_{T+1}=\hat{y}_{T+1}^{\prime} * I_{1}
$$


or in the additive form

$$
\hat{y}_{T+1}=\hat{y}_{T+1}^{\prime}+I_{1}
$$

The applicability of Equations (9) and (10) is determined by many factors, such as the real dataset, the proposed forecast models and the evaluation methods. Numerical experiments and simulations generally provide reliable ways to validate their applicability, as shown in Section 4.1.

\subsection{Adaptive Coefficient Method}

Forecasting techniques based on trend extrapolation include the moving average procedure, the exponential smoothing model and the adaptive coefficient method. Specifically, the moving average procedure assigns equal weights to the $N-1$ historical data and the current value, where $N$ is the moving average length [26]. The basic exponential smoothing model exerts exponentially decreasing weights on all data points that are controlled by the constant smoothing parameter between 0 and 1 [27]. The adaptive coefficient method, or adaptive exponential smoothing, was developed on the basis of the exponential smoothing model, with the smoothing parameter changing adaptively according to forecast errors $[2,28]$. The following schemes are the first- and second-order adaptive coefficient methods.

For a time $t$, using the first-order adaptive coefficient method, denote the forecasted value for time $t+1$ as $\hat{x}_{t+1}$. Suppose we have $\alpha_{t}$ varying with time $t, \hat{x}_{t+1}$ is the weighted average of the observed data $x_{t}$ and the forecasted value $\hat{x}_{t}$. which indicates that

$$
\hat{x}_{t+1}=\alpha_{t} x_{t}+\left(1-\alpha_{t}\right) \hat{x}_{t}=\hat{x}_{t}+\alpha_{t}\left(x_{t}-\hat{x}_{t}\right)=\hat{x}_{t}+\alpha_{t} e_{t}
$$

where $e_{t}$ is the forecasting error at time $t$.

Introduce a constant $\beta(0<\beta<1)$ and generate the exponential smoothing sequence of $e_{k}(k=1,2, \cdots, t)$ to reflect the systematic error $E_{t}$ during the time period $t$, namely,

$$
E_{t}=\beta e_{t}+\beta(1-\beta) e_{t-1}+\cdots+\beta(1-\beta)^{t-1} e_{1}=\beta e_{t}+(1-\beta) E_{t-1}
$$

To make sure that $\alpha_{t}$ always varies between 0 and 1 , define $M_{t}$ as

$$
M_{t}=\beta\left|e_{t}\right|+\beta(1-\beta)\left|e_{t-1}\right|+\cdots+\beta(1-\beta)^{t-1}\left|e_{1}\right|=\beta\left|e_{t}\right|+(1-\beta) M_{t-1}
$$

Then, the first-order adaptive coefficient $\alpha_{t}$ is calculated as

$$
\alpha_{t}=\left|E_{t}\right| / M_{t}
$$

The aforementioned formulae outline the basic algorithem of the first-order adaptive coefficient method. Based on the first-order adaptive coefficient method, two additional variables are introduced in the second-order adaptive coefficient technique, which are represented as follows:

$$
\begin{gathered}
S_{t}^{(1)}=\alpha_{t} x_{t}+\left(1-\alpha_{t}\right) S_{t-1}^{(1)} \\
S_{t}^{(2)}=\alpha_{t} S_{t}^{(1)}+\left(1-\alpha_{t}\right) S_{t-1}^{(2)}
\end{gathered}
$$

where $\alpha_{t}$ is computed according to Equation (14). The forecasted value of the second-order adaptive coefficient approach is calculated as follows:

$$
\hat{x}_{t+1}=\hat{a}_{t}+\hat{b}_{t}
$$

where

$$
\hat{a}_{t}=2 S_{t}^{(1)}-S_{t}^{(2)}
$$




$$
\hat{b}_{t}=\frac{\alpha_{t}}{1-\alpha_{t}}\left[S_{t}^{(1)}-S_{t}^{(2)}\right]
$$

It is worth noting that in both the first- and the second-order adaptive coefficient approaches, $\beta$ has a significant effect on final forecasting performance. Only the parameter $\beta$ need to be selected prior to launching the algorithm and other parameters are calculated via iterations. However, with respect to conventional adaptive coefficient models, the value of $\beta$ is subjectively predetermined. Consequently, this paper employs the cuckoo search algorithm to improve conventional adaptive coefficient models by optimizing the value of $\beta$. By minimizing the objective function of fitting errors, this metaheuristic algorithm provides a solid foundation for the selection of the $\beta$ value and results in improved forecasting accuracy, as demonstrated in Section 3.

\subsection{Cuckoo Search Algorithm (CS)}

Proposed in 2009 by Yang and Deb [29], the cuckoo search algorithm is based on the obligate brood parasitism of some cuckoo species, in combination with the Lévy flights, rather than simple isotropic random walks [26]. It is one of the latest metaheuristic algorithms inspired by nature. For detailed information about cuckoo breeding behavior and their aggressive reproduction strategy, readers can refer to [30].

Three idealized rules [30] of the cuckoo search algorithm for simple and single optimization include: (1) each cuckoo lays one egg at a time and put it in a randomly chosen host nest; (2) the nest that consists of the most high-quality eggs will be carried over to the next generations; (3) the egg to be discovered by the host bird has a probability $p_{a} \in[0,1]$ and the number of available host nests is fixed. For multi-objective optimization, these basic rules should be modified as introduced in [31].

For the convenience of practical implementation, Yang and Deb [30] suppose that each egg in a nest denotes a solution, and the aim is to replace the not-so-good solutions in the nests by the new and potentially better solutions (cuckoos). To generate a new solution $x_{i}^{(t+1)}$ for a cuckoo $i$, a Lévy flight is performed:

$$
x_{i}^{(t+1)}=x_{i}^{(t)}+\alpha \oplus \operatorname{Lev} y(\lambda)
$$

where $\alpha>0$ is the step size and the product $\oplus$ indicates entry-wise multiplications [26]. And the length of the random step of Lévy flight is drawn from the Lévy distribution with both mean and variance infinite:

$$
\text { Levy } \sim \mu=t^{-\lambda},(1<\lambda \leq 3)
$$

The interested readers can refer to $[28,29]$ for more details of the cuckoo search algorithm.

\section{Proposed Model}

This paper proposes four hybrid forecasting models, which include $A-F A C-C S, M-F A C-C S$, $A-S A C-C S$, and $M-S A C-C S$. The four hybrid forecasting models have the following three steps.

- Step 1. The original dataset is processed by the seasonal exponential adjustment technique to remove periodic fluctuations

- $\quad$ Step 2. The trend series are regarded as input sets of the adaptive coefficient forecasting model.

- Step 3. The exogenous variable $\beta$ of the adaptive coefficient method is optimized by the cuckoo search algorithm instead of being subjectively predetermined.

Considering that the seasonal exponential adjustment technique has both multiplicative and additive form, and the adaptive coefficient forecasting model can be divided into first-order and second-order. So the four hybrid models are listed in the following, and the flowchart of these four hybrid models is illustrated in Figure 1.

- (a) The $A-F A C-C S$ model is the one with additively seasonal exponential adjustment $(A)$, and $C S$ optimizes the first-order adaptive coefficient model ( $F A C)$. 
- (b) The M-FAC-CS model is the one with multiplicative seasonal exponential adjustment $(M)$, and $C S$ optimizes the first-order adaptive coefficient model (FAC).

- (c) The $A-S A C-C S$ model is the one with additive seasonal exponential adjustment $(A)$, and $C S$ optimizes the second-order adaptive coefficient model ( $S A C)$.

- (d) The M-SAC-CS model is the one with multiplicative seasonal exponential adjustment $(M)$, and $C S$ optimizes the second-order adaptive coefficient model ( $S A C)$.

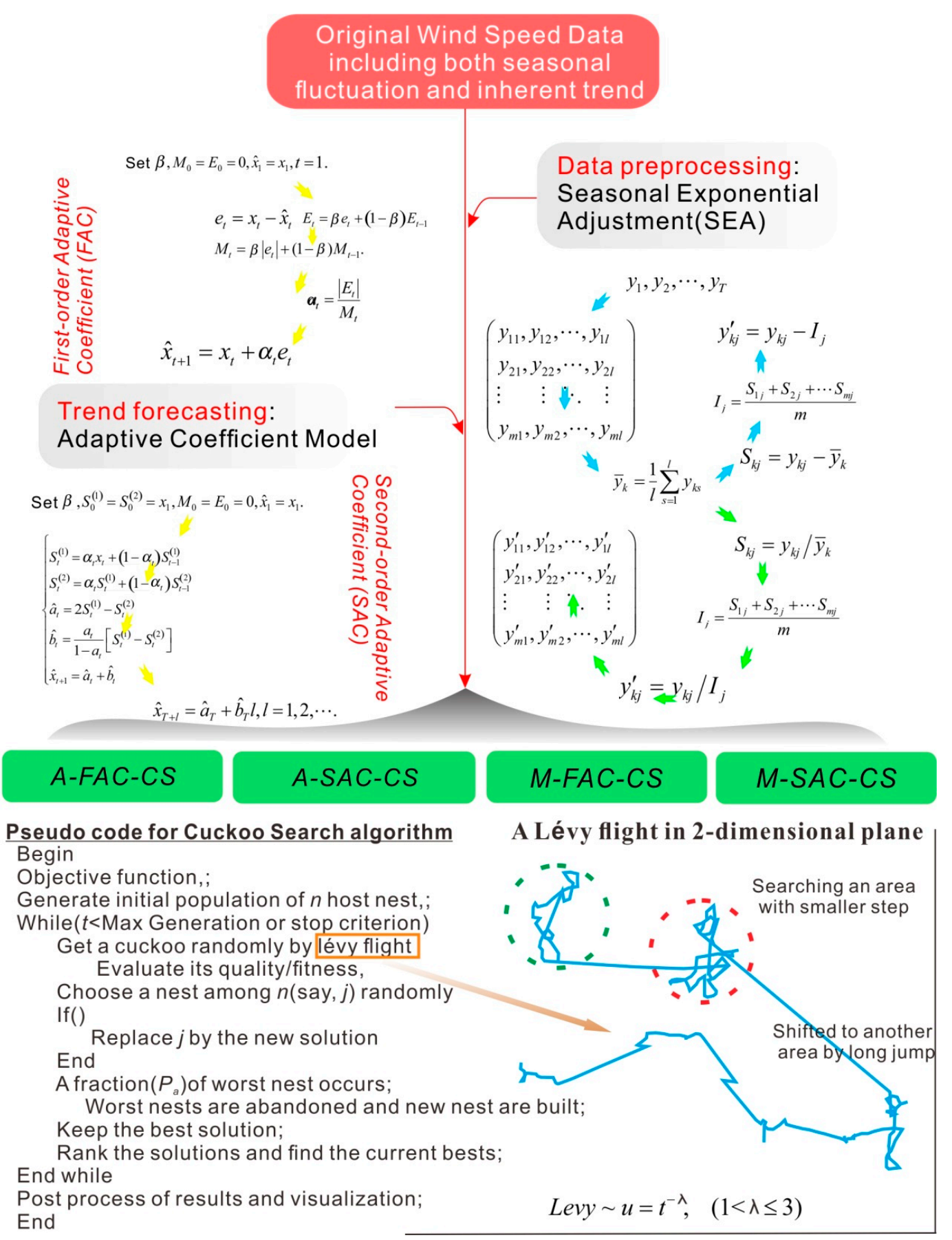

Figure 1. The flowchart of the developed hybrid forecasting models. 


\section{Simulation Results and Discussion}

The daily average wind-speed data is collected from four observation sites of the Xinjiang Uygur Autonomous Region located in northwestern China, including Urumqi City, Korla City, Altay Region and Hami Region. The sample period covers more than four years, from 1 January 2009 to 18 September 2013. The original wind-speed time series of the four observation sites are plotted in Figure 2 together with detailed geographic information.

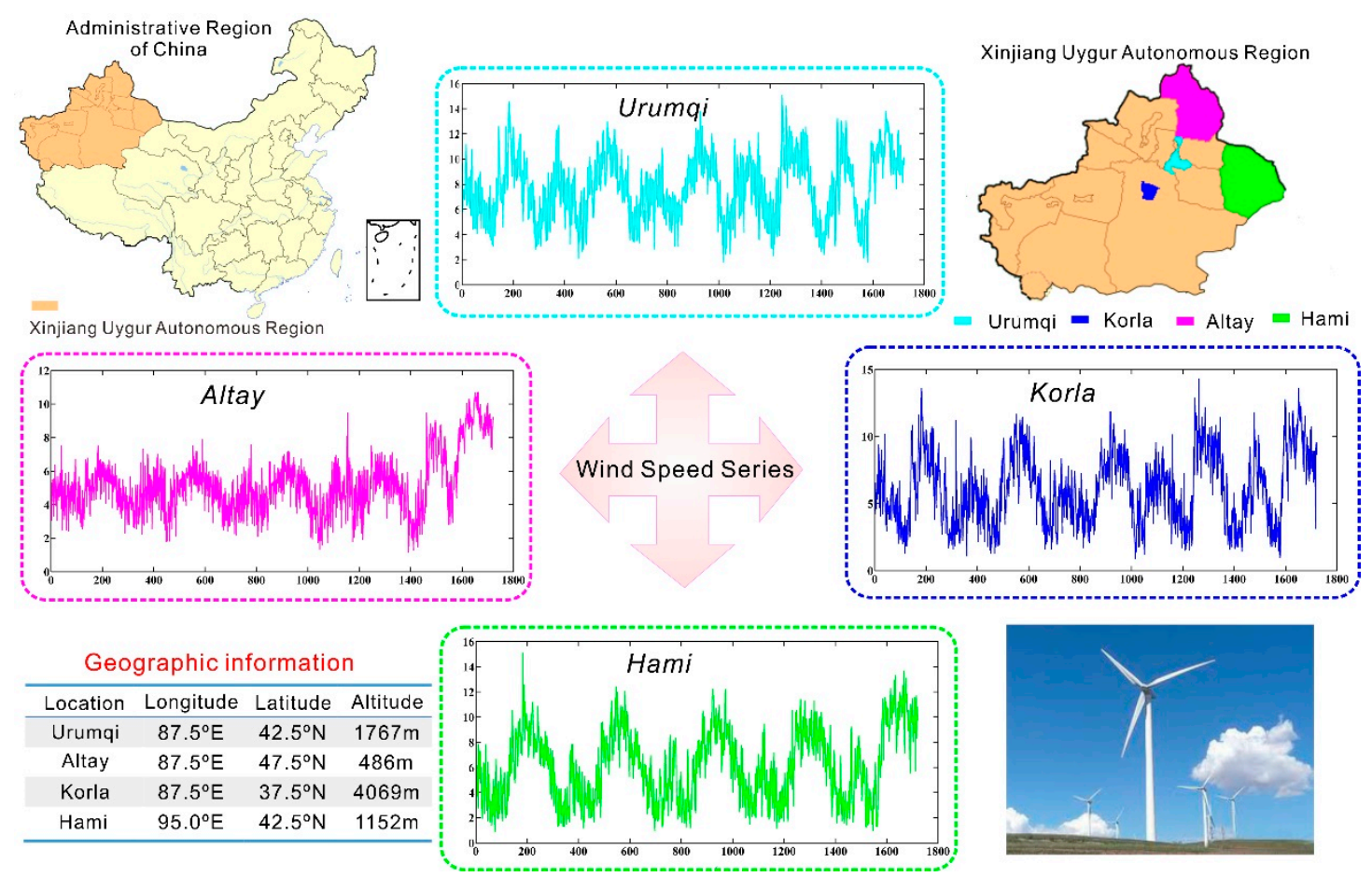

Figure 2. Wind-speed time series and related geographic information.

In Figure 2, the number of the day without unit is in the horizontal axis, while the vertical axis is the wind speed with unit m/s. As we can see from the line charts in Figure 2, for the four observation sites, there are some yearly periodic components in the original wind-speed series. Thus we set the cycle length to be one year by means of direct observation from Figure 2. In particular, original data from 1 January 2009 to 31 December 2012 consisting of four cycles are utilized to calculate the seasonal indices through seasonal exponential adjustment technique as introduced in Section 2.1. In order to validate the effectiveness of the proposed four hybrid forecasting models, daily wind speeds from 1 January 2013 to 31 August 2013 are applied to forecast one day ahead wind-speed data. To facilitate the evaluation and comparison process, the forecast results are summarized according to different forecast months, and two main error criteria are calculated as follows to reflect integral forecasting performance:

$$
\begin{gathered}
\text { MAPE }=\frac{1}{N} \sum_{i=1}^{N}\left|\frac{y_{i}-\hat{y}_{i}}{y_{i}}\right| \times 100 \% \\
\text { RMSE }=\sqrt{\frac{1}{N} \sum_{i=1}^{N}\left(y_{i}-\hat{y}_{i}\right)^{2}}
\end{gathered}
$$

where $N$ is the forecasting periods number, $y_{i}$ is the true value at time $i$ and $\hat{y}_{i}$ is the forecasted value corespondinly. 
Next we take Urumqi as the representative example to demonstrate the presented hybrid forecasting methods step by step with intermediary outcomes. In Figure 3, the number of the day without unit is in the horizontal axis, and the vertical axis is the wind speed with unit $\mathrm{m} / \mathrm{s}$. As illustrated in Figure 3, the trend series obtained from multiplicative and additive seasonal exponential adjustment are depicted respectively in Figure 3 part A and Figure 3 part B in contrast with the original wind-speed series of Urumqi. In addition, mean values and standard deviations of the above three wind-speed time series are calculated as listed in the table of Figure 3 part D. The vivid line charts and computed results indicate that the seasonal exponential adjustment technique contributes to eliminating periodic fluctuations and lowering standard deviations. That is to say, the trend series are to some extent easier to forecast than the original series.
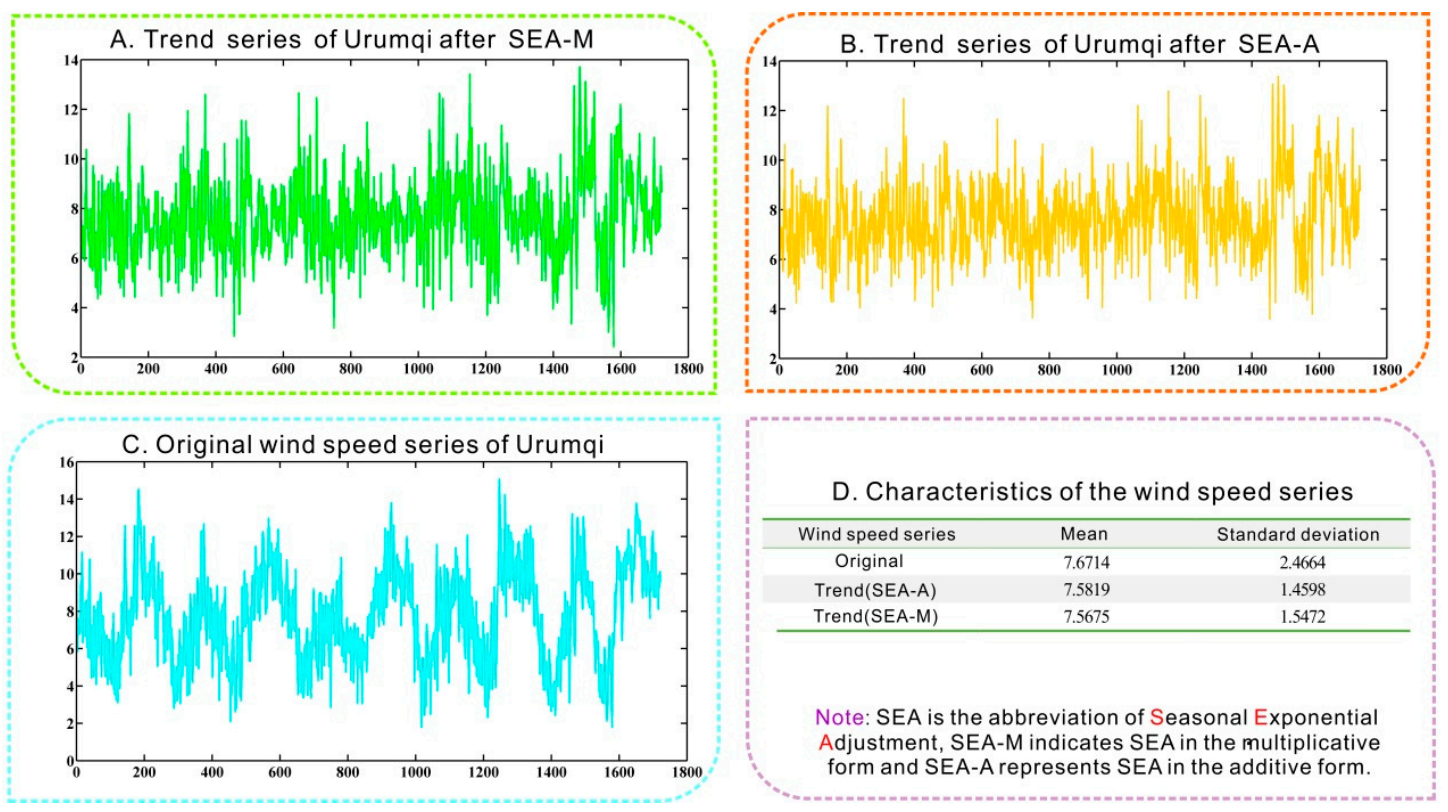

Figure 3. The trend series after seasonal exponential adjustment technique.

\subsection{Comparisons of Four Hybrid Models}

The parameters of the hybrid models for the cuckoo search algorithm are as follows. There are 25 nests, the step size $\alpha$ is 1 , the Lévy flight parameter $\lambda$ is 1.5 , the discovery rate of alien eggs $p_{a}$ is 0.25 , and there are 1000 iterations in total. As demonstrated in Figure 4, we plot the forecasting as well as the actual values of Urumqi from January to August in 2013, respectively, under the four developed hybrid forecasting models, namely, $A-F A C-C S, A-S A C-C S, M-F A C-C S$ and $M-S A C-C S$, as defined in Section 2.3. Moreover, the forecasting errors of the four different forecasting strategies, including both $M A P E$ (mean absolute percentage error) and RMSE (root mean square error) criteria, are also presented in the table of Figure 4. In Figure 4, the number of the day without unit is in the horizontal axis and the vertical axis is the wind speed with unit $\mathrm{m} / \mathrm{s}$. 


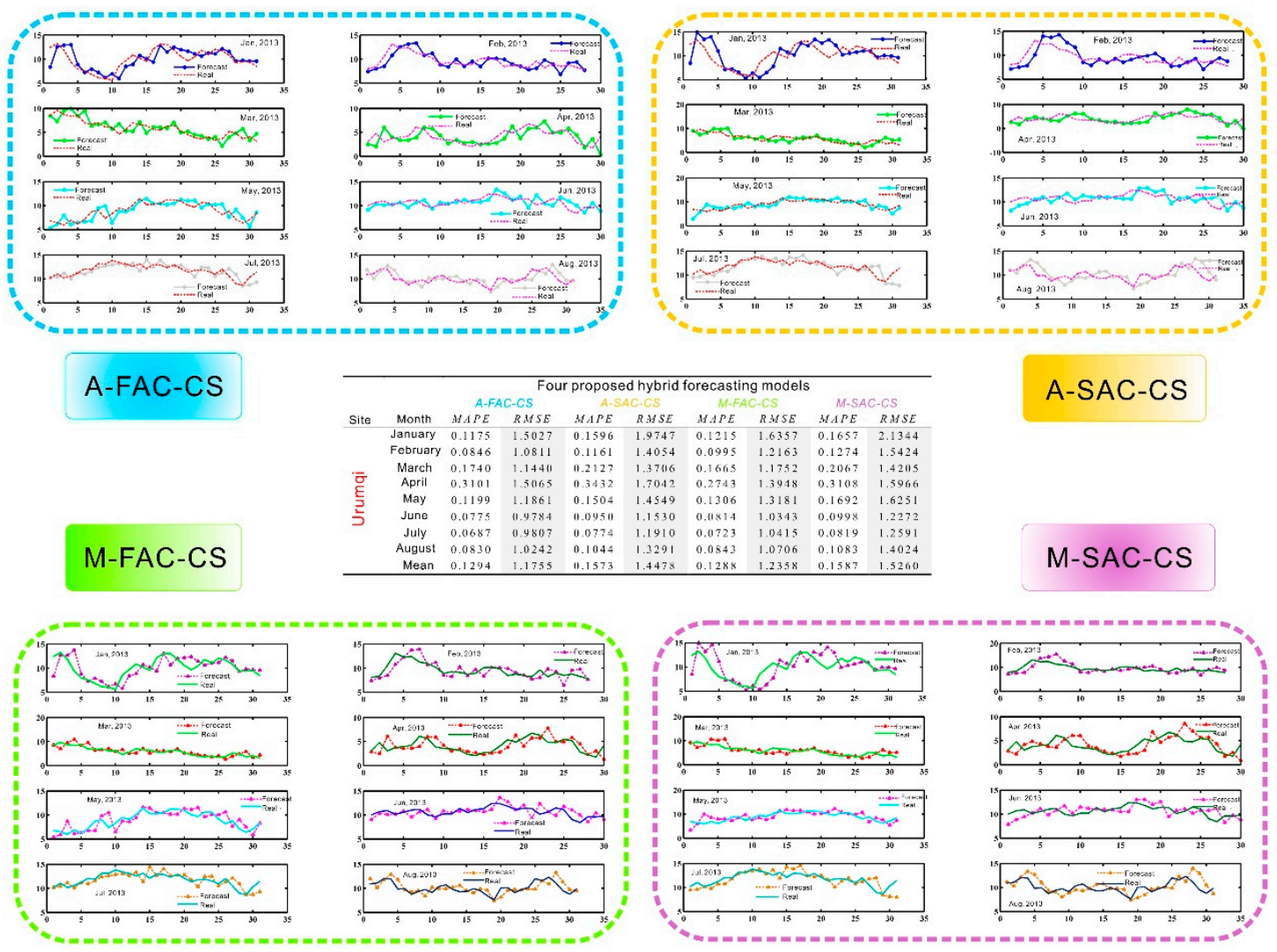

Figure 4. Forecasting results of Urumqi from January to August, 2013.

We can vividly see that the developed hybrid forecasting procedures are capable of achieving satisfactory forecasting performance with respect to the Urumqi observation site. In particular, $M-F A C-C S$ leads to the lowest mean MAPE value of $12.88 \%$, while $A-F A C-C S$ results in the smallest mean RMSE value of 1.1755. Therefore, we can obtain the following conclusion: the first-order adaptive coefficient method performs much better than the second-order adaptive coefficient model, regardless of error criteria, which indicates that higher order algorithms do not necessarily contribute to more desirable forecasting accuracy.

Table 1 lists the forecasting errors of the four kinds of hybrid models, namely A-FAC-CS, A-SAC-CS, $M-F A C-C S$ and M-SAC-CS, from January to August 2013 with respect to four targeted wind-speed observation sites, including Urumqi, Altay, Korla as well as Hami. Moreover, for each observation site, the average MAPE and RMSE values of the eight months are also calculated for each of the presented hybrid forecasting models to reflect and evaluate the overall forecasting performance.

Table 1. Forecast errors of the developed hybrid models.

\begin{tabular}{|c|c|c|c|c|c|c|c|c|c|}
\hline \multirow{3}{*}{ Site } & \multirow{3}{*}{ Month } & \multicolumn{2}{|c|}{$A-F A C-C S$} & \multicolumn{2}{|c|}{$A-S A C-C S$} & \multicolumn{2}{|c|}{$M-F A C-C S$} & \multicolumn{2}{|c|}{$M-S A C-C S$} \\
\hline & & MAPE & RMSE & MAPE & RMSE & MAPE & RMSE & MAPE & RMSE \\
\hline & & $(\%)$ & $(\mathrm{m} / \mathrm{s})$ & $(\%)$ & $(\mathrm{m} / \mathrm{s})$ & $(\%)$ & $(\mathrm{m} / \mathrm{s})$ & $(\%)$ & $(\mathrm{m} / \mathrm{s})$ \\
\hline \multirow{9}{*}{ Urumqi } & Jan. & 11.75 & 1.5027 & 15.96 & 1.9747 & 12.15 & 1.6357 & 16.57 & 2.1344 \\
\hline & Feb. & 8.46 & 1.0811 & 11.61 & 1.4054 & 9.95 & 1.2163 & 12.74 & 1.5424 \\
\hline & Mar. & 17.40 & 1.1440 & 21.27 & 1.3706 & 16.65 & 1.1752 & 20.67 & 1.4205 \\
\hline & Apr. & 31.01 & 1.5065 & 34.32 & 1.7042 & 27.43 & 1.3948 & 31.08 & 1.5966 \\
\hline & May. & 11.99 & 1.1861 & 15.04 & 1.4549 & 13.06 & 1.3181 & 16.92 & 1.6251 \\
\hline & Jun. & 7.75 & 0.9784 & 9.50 & 1.1530 & 8.14 & 1.0343 & 9.98 & 1.2272 \\
\hline & Jul. & 6.87 & 0.9807 & 7.74 & 1.1910 & 7.23 & 1.0415 & 8.19 & 1.2591 \\
\hline & Aug. & 8.30 & 1.0242 & 10.44 & 1.3291 & 8.43 & 1.0706 & 10.83 & 1.4024 \\
\hline & Mean & 12.94 & 1.1755 & 15.73 & 1.4478 & 12.88 & 1.2358 & 15.87 & 1.5260 \\
\hline
\end{tabular}


Table 1. Cont.

\begin{tabular}{|c|c|c|c|c|c|c|c|c|c|}
\hline \multirow{3}{*}{ Site } & \multirow{3}{*}{ Month } & \multicolumn{2}{|c|}{$A-F A C-C S$} & \multicolumn{2}{|c|}{$A-S A C-C S$} & \multicolumn{2}{|c|}{$M-F A C-C S$} & \multicolumn{2}{|c|}{$M-S A C-C S$} \\
\hline & & MAPE & RMSE & MAPE & RMSE & MAPE & RMSE & MAPE & RMSE \\
\hline & & $(\%)$ & $(\mathrm{m} / \mathrm{s})$ & $(\%)$ & $(\mathrm{m} / \mathrm{s})$ & $(\%)$ & $(\mathrm{m} / \mathrm{s})$ & $(\%)$ & $(\mathrm{m} / \mathrm{s})$ \\
\hline \multirow{9}{*}{ Altay } & Jan. & 17.33 & 1.7730 & 17.53 & 1.7924 & 18.55 & 1.7958 & 19.35 & 1.8774 \\
\hline & Feb. & 12.77 & 1.1273 & 13.92 & 1.2158 & 15.65 & 1.3816 & 17.54 & 1.5096 \\
\hline & Mar. & 21.90 & 1.3127 & 22.00 & 1.3170 & 21.66 & 1.3692 & 22.46 & 1.4189 \\
\hline & Apr. & 23.49 & 1.2979 & 22.31 & 1.2727 & 24.18 & 1.3401 & 22.14 & 1.2667 \\
\hline & May. & 13.81 & 1.2823 & 14.61 & 1.3661 & 13.52 & 1.2314 & 14.62 & 1.3338 \\
\hline & Jun. & 6.72 & 0.7783 & 6.95 & 0.8191 & 8.02 & 0.9390 & 9.24 & 1.0490 \\
\hline & Jul. & 6.76 & 0.7611 & 6.95 & 0.7728 & 8.42 & 0.9406 & 8.23 & 0.9445 \\
\hline & Aug. & 7.40 & 0.7614 & 9.10 & 0.9186 & 9.35 & 0.9420 & 9.91 & 0.9939 \\
\hline & Mean & 13.77 & 1.1367 & 14.17 & 1.1843 & 14.92 & 1.2425 & 15.44 & 1.2992 \\
\hline \multirow{9}{*}{ Korla } & Jan. & 18.43 & 1.5578 & 31.84 & 2.5856 & 19.99 & 1.8015 & 27.01 & 2.4067 \\
\hline & Feb. & 18.86 & 1.5369 & 17.67 & 1.7954 & 22.12 & 1.8093 & 27.14 & 2.3944 \\
\hline & Mar. & 24.55 & 1.2167 & 37.71 & 1.6057 & 25.38 & 1.2436 & 35.48 & 1.6143 \\
\hline & Apr. & 37.61 & 1.1623 & 44.25 & 1.2791 & 35.30 & 1.0951 & 42.02 & 1.1497 \\
\hline & May. & 17.22 & 1.5213 & 22.25 & 1.9241 & 19.45 & 1.7472 & 22.34 & 1.9787 \\
\hline & Jun. & 8.47 & 1.0407 & 13.65 & 1.6622 & 9.05 & 1.1248 & 14.82 & 1.6642 \\
\hline & Jul. & 9.88 & 1.2926 & 12.94 & 1.6240 & 10.65 & 1.4113 & 12.45 & 1.5789 \\
\hline & Aug. & 9.63 & 1.0526 & 15.75 & 1.6861 & 9.99 & 1.0897 & 13.91 & 1.5363 \\
\hline & Mean & 18.08 & 1.2976 & 24.51 & 1.7703 & 18.99 & 1.4153 & 24.39 & 1.7904 \\
\hline \multirow{9}{*}{ Hami } & Jan. & 28.28 & 1.4712 & 26.40 & 1.5940 & 25.53 & 1.5595 & 27.28 & 1.9086 \\
\hline & Feb. & 21.78 & 1.1774 & 26.23 & 1.4843 & 24.55 & 1.4277 & 30.87 & 1.6943 \\
\hline & Mar. & 43.75 & 1.3477 & 49.15 & 1.5802 & 43.84 & 1.3548 & 48.86 & 1.6425 \\
\hline & Apr. & 30.55 & 1.2230 & 31.96 & 1.3845 & 30.19 & 1.3349 & 33.30 & 1.5001 \\
\hline & May. & 11.77 & 1.5093 & 14.66 & 1.9230 & 14.79 & 1.9089 & 18.98 & 2.5150 \\
\hline & Jun. & 10.34 & 1.2545 & 11.26 & 1.4036 & 11.02 & 1.3574 & 12.43 & 1.6215 \\
\hline & Jul. & 10.93 & 1.5589 & 14.23 & 2.0656 & 11.20 & 1.5852 & 14.39 & 2.0678 \\
\hline & Aug. & 15.71 & 1.9203 & 16.53 & 2.0228 & 16.28 & 1.9700 & 17.03 & 2.0526 \\
\hline & Mean & 21.64 & 1.4328 & 23.80 & 1.6823 & 22.17 & 1.5623 & 25.39 & 1.8753 \\
\hline
\end{tabular}

From Table 1, it can be concluded that for the observation sites of Altay, Korla and Hami, the presented $A-F A C-C S$ forecasting strategy can get the most satisfactory forecasting accuracy. In particular, the mean MAPE and RMSE values of Altay are 13.77\% and 1.1367, while for Korla they are $18.08 \%$ and 1.2976 , respectively. As for Hami, the forecast errors rise slightly to $21.64 \%$ and 1.4328 . In addition, numerical experiments and simulation results of the four observation sites suggest that the additive seasonal exponential adjustment technique outperforms the multiplicative one when the average forecast errors of the two pairs, namely $A-F A C-C S$ and $M-F A C-C S, A-S A C-C S$ and $M-S A C-C S$, are compared. Similarly, intensive comparisons of $A-F A C-C S$ and $A-S A C-C S$ in addition to M-FAC-CS and $M-S A C-C S$ indicate that the first-order adaptive coefficient method leads to much more accurate forecasting results than the second-order adaptive coefficient model.

\subsection{Compared with Classic Individual Models}

In this section, to forecast the daily mean wind speed of the four observation sites as mentioned on Section 3, we also apply four individual models. The models are back propagation $(B P)$ neural network (via Neural Network Toolbox of MATLAB), the autoregressive integrated moving average (ARIMA) model (via Eviews 7), the single first-order adaptive coefficient (FAC) method and the single second-order adaptive coefficient $(S A C)$ method. Those methods are compared with the hybrid model in terms of forecasting ability. Note that the parameters $\beta$ for both FAC and SAC are set as 0.2. The forecast errors of the four conventional approaches are shown in Table 2 with respect to the monthly and average MAPE and RMSE values. 
Table 2. Forecast errors of the individual forecasting models.

\begin{tabular}{|c|c|c|c|c|c|c|c|c|c|}
\hline \multirow{3}{*}{ Site } & \multirow{3}{*}{ Month } & \multicolumn{2}{|c|}{$B P$} & \multicolumn{2}{|c|}{ ARIMA } & \multicolumn{2}{|c|}{ FAC } & \multicolumn{2}{|c|}{$S A C$} \\
\hline & & $M A P E$ & RMSE & MAPE & RMSE & MAPE & RMSE & MAPE & RMSE \\
\hline & & $(\%)$ & $(\mathrm{m} / \mathrm{s})$ & $(\%)$ & $(\mathrm{m} / \mathrm{s})$ & $(\%)$ & $(\mathrm{m} / \mathrm{s})$ & $(\%)$ & $(\mathrm{m} / \mathrm{s})$ \\
\hline \multirow{9}{*}{ Urumqi } & Jan. & 29.18 & 3.5627 & 20.98 & 2.4513 & 27.90 & 3.5156 & 20.58 & 2.8465 \\
\hline & Feb. & 23.75 & 2.7678 & 10.87 & 1.5316 & 11.14 & 1.7930 & 18.04 & 1.7933 \\
\hline & Mar. & 39.43 & 2.1332 & 71.11 & 3.5379 & 51.44 & 2.6670 & 57.09 & 3.0677 \\
\hline & Apr. & 91.88 & 3.1666 & 32.62 & 1.4003 & 73.91 & 2.6822 & 33.24 & 1.5404 \\
\hline & May. & 20.87 & 2.4769 & 43.56 & 4.4628 & 26.58 & 2.8222 & 36.24 & 3.6641 \\
\hline & Jun. & 28.58 & 3.2256 & 15.03 & 1.8523 & 23.62 & 2.8243 & 13.17 & 1.6420 \\
\hline & Jul. & 30.88 & 3.9388 & 14.41 & 2.0055 & 12.73 & 1.7973 & 10.05 & 1.4265 \\
\hline & Aug. & 17.49 & 2.1202 & 13.81 & 1.4732 & 10.39 & 1.2257 & 16.51 & 1.7810 \\
\hline & Mean & 35.26 & 2.9240 & 27.80 & 2.3394 & 29.71 & 2.4159 & 25.62 & 2.2202 \\
\hline \multirow{9}{*}{ Altay } & Jan. & 40.43 & 3.2895 & 37.19 & 3.0736 & 36.33 & 3.0387 & 33.90 & 2.8755 \\
\hline & Feb. & 38.38 & 3.1163 & 9.59 & 0.8608 & 16.99 & 1.6680 & 12.13 & 1.0236 \\
\hline & Mar. & 25.38 & 1.6930 & 40.31 & 2.2419 & 24.70 & 1.4365 & 34.39 & 1.9389 \\
\hline & Apr. & 18.40 & 1.0063 & 18.64 & 1.0948 & 21.30 & 1.1334 & 34.28 & 1.7427 \\
\hline & May. & 26.79 & 2.3141 & 27.82 & 2.3983 & 17.79 & 1.6172 & 18.83 & 1.6681 \\
\hline & Jun. & 28.33 & 2.6634 & 12.50 & 1.2670 & 8.78 & 1.0173 & 14.23 & 1.5739 \\
\hline & Jul. & 26.50 & 2.7111 & 8.74 & 1.0413 & 6.42 & 0.7566 & 6.60 & 0.7798 \\
\hline & Aug. & 13.69 & 1.3585 & 8.00 & 0.8084 & 7.05 & 0.6831 & 10.70 & 1.0428 \\
\hline & Mean & 27.24 & 2.2690 & 20.35 & 1.5983 & 17.42 & 1.4188 & 20.63 & 1.5807 \\
\hline \multirow{9}{*}{ Korla } & Jan. & 33.98 & 2.8433 & 38.10 & 2.4806 & 31.82 & 2.9070 & 28.30 & 2.4524 \\
\hline & Feb. & 23.81 & 2.0919 & 10.15 & 1.0991 & 14.44 & 1.4556 & 17.79 & 1.4202 \\
\hline & Mar. & 46.62 & 1.6986 & 88.11 & 2.9736 & 63.89 & 2.2589 & 66.56 & 2.4696 \\
\hline & Apr. & 120.12 & 2.6185 & 42.88 & 1.0565 & 82.55 & 1.9666 & 37.12 & 1.0090 \\
\hline & May. & 32.66 & 3.7806 & 29.87 & 3.4455 & 36.94 & 3.7943 & 43.35 & 4.1106 \\
\hline & Jun. & 32.33 & 3.4578 & 12.95 & 1.6919 & 20.56 & 2.5124 & 14.01 & 1.5748 \\
\hline & Jul. & 37.08 & 4.3698 & 36.48 & 4.2827 & 12.75 & 1.6153 & 13.17 & 1.6535 \\
\hline & Aug. & 19.13 & 2.2059 & 18.60 & 1.7018 & 13.98 & 1.3956 & 22.53 & 2.0864 \\
\hline & Mean & 43.22 & 2.8833 & 34.64 & 2.3415 & 34.62 & 2.2382 & 30.35 & 2.0971 \\
\hline \multirow{9}{*}{ Hami } & Jan. & 50.96 & 2.1572 & 41.63 & 2.0081 & 37.87 & 2.3491 & 41.12 & 2.8150 \\
\hline & Feb. & 31.59 & 1.4353 & 57.07 & 2.0690 & 31.78 & 1.4446 & 34.35 & 1.5961 \\
\hline & Mar. & 95.89 & 2.1456 & 54.76 & 1.4215 & 75.49 & 1.7488 & 89.69 & 2.0288 \\
\hline & Apr. & 33.35 & 1.3148 & 55.19 & 2.8800 & 50.70 & 2.0461 & 55.29 & 2.0860 \\
\hline & May. & 57.16 & 5.8094 & 31.80 & 3.4077 & 37.40 & 4.1542 & 25.85 & 3.2662 \\
\hline & Jun. & 47.21 & 5.3752 & 14.45 & 1.8675 & 17.78 & 2.0651 & 14.55 & 1.8092 \\
\hline & Jul. & 37.52 & 4.3132 & 12.15 & 1.6135 & 8.75 & 1.2439 & 8.75 & 1.2199 \\
\hline & Aug. & 25.37 & 2.9380 & 19.59 & 2.1095 & 14.50 & 1.5831 & 17.68 & 1.9387 \\
\hline & Mean & 47.38 & 3.1861 & 35.83 & 2.1721 & 34.28 & 2.0794 & 35.91 & 2.0950 \\
\hline
\end{tabular}

As shown in Table 2, the mean MAPE values of the BP neural network regarding the four observation sites, namely Urumqi, Altay, Korla as well as Hami, are $35.26 \%, 27.24 \%, 43.22 \%$ and $47.38 \%$, respectively, while the average RMSE values of the ARIMA model are 2.3394, 1.5983, 2.3415 and 2.1721. In addition, the $B P$ neural network and the ARIMA model yield extremely high forecast errors for some months. For example, the $M A P E$ value of the $B P$ neural network with respect to Urumqi in April is as high as $91.88 \%$ and the MAPE value of the ARIMA model with respect to Korla in March reaches up to $88.11 \%$. Apparently, these extremely inaccurate forecasting results have an adverse effect on wind farm operations and may threaten the security of the grid when wind power is integrated for electricity supply. Thus, it is highly significant to propose novel hybrid forecasting strategies to replace the individual forecasting models in practice and achieve desirable forecasting accuracy.

To better illustrate the superiority of the hybrid forecasting approaches presented in this paper, we list the mean MAPE values from January to August in 2013 obtained from the four individual forecasting models as well as one hybrid forecasting strategy in Table 3. Note that there is no harm in 
selecting the specific hybrid model with the maximal MAPE value among the four kinds of developed hybrid methods to represent the hybrid forecasting models in the last column of Table 3.

Table 3. The mean MAPE values (\%) of different forecasting models.

\begin{tabular}{cccccc}
\hline Site & $\boldsymbol{B P}$ & ARIMA & $\boldsymbol{F A C}$ & $\boldsymbol{S A C}$ & Hybrid \\
\hline Urumqi & 35.26 & 27.80 & 29.71 & 25.62 & 15.87 \\
Altay & 27.24 & 20.35 & 17.42 & 20.63 & 15.44 \\
Korla & 43.22 & 34.64 & 34.62 & 30.35 & 24.51 \\
Hami & 47.38 & 35.83 & 34.28 & 35.91 & 25.39 \\
\hline
\end{tabular}

As we can clearly see from Table 3, the presented hybrid forecasting models can remarkably reduce forecasting errors compared with conventional methods. Taking Urumqi as an example, through simple calculation we find the mean MAPE value using the hybrid model decrease by $19.39 \%, 11.93 \%$, $13.84 \%$ and $7.75 \%$ compared to the BP neural network, ARIMA, FAC and SAC models, respectively. Similar results can be made based on the forecast results of other three observation sites as illustrated in Table 3. To sum up, the novel hybrid forecasting strategies developed in this paper are effective, which is proven by a number of numerical simulations whose results are listed in Tables 1 and 2 . Furthermore, the innovative hybrid models make significant improvements to traditional single forecasting approaches and provide more satisfactory forecasting accuracy for practical applications in wind power generation and grid regulation.

Next, to verify the significance of the parameter optimization process in the hybrid forecasting strategies, we construct four kinds of forecasting approaches using the seasonal exponential adjustment technique and the adaptive coefficient methods whose exogenous parameters $\beta$ are predetermined (with the value 0.2 ) in the conventional way rather than being optimized by the cuckoo search algorithm. These four kinds of methods are denoted as $A-F A C, A-S A C, M-F A C$ and $M-S A C$, respectively, corresponding to their counterparts $A-F A C-C S, A-S A C-C S, M-F A C-C S$ and $M-S A C-C S$ with optimal parameters attained from the cuckoo search algorithm, respectively. Furthermore, their forecast errors are summarized in Table 4.

Table 4. Forecast errors of the hybrid models with predetermined parameters.

\begin{tabular}{|c|c|c|c|c|c|c|c|c|c|}
\hline \multirow{3}{*}{ Site } & \multirow{3}{*}{ Month } & \multicolumn{2}{|c|}{$A-F A C$} & \multicolumn{2}{|c|}{$A-S A C$} & \multicolumn{2}{|c|}{$M-F A C$} & \multicolumn{2}{|c|}{$M-S A C$} \\
\hline & & MAPE & RMSE & MAPE & RMSE & MAPE & RMSE & MAPE & RMSE \\
\hline & & $(\%)$ & $(\mathrm{m} / \mathrm{s})$ & $(\%)$ & $(\mathrm{m} / \mathrm{s})$ & $(\%)$ & $(\mathrm{m} / \mathrm{s})$ & $(\%)$ & $(\mathrm{m} / \mathrm{s})$ \\
\hline \multirow{9}{*}{ Urumqi } & Jan. & 22.80 & 2.9656 & 21.07 & 2.9327 & 23.28 & 3.0388 & 20.62 & 2.9587 \\
\hline & Feb. & 11.15 & 1.7389 & 10.96 & 1.4012 & 11.82 & 1.8162 & 11.54 & 1.4282 \\
\hline & Mar. & 37.45 & 2.0957 & 46.76 & 2.5729 & 37.19 & 2.1349 & 46.36 & 2.6037 \\
\hline & Apr. & 52.60 & 1.9398 & 36.64 & 1.6318 & 50.31 & 1.8568 & 37.08 & 1.5703 \\
\hline & May. & 23.06 & 2.3548 & 27.38 & 2.6666 & 23.92 & 2.4360 & 28.64 & 2.7845 \\
\hline & Jun. & 10.98 & 1.3755 & 9.88 & 1.3285 & 11.18 & 1.4089 & 10.12 & 1.3996 \\
\hline & Jul. & 10.84 & 1.5237 & 10.95 & 1.5242 & 11.08 & 1.5498 & 11.75 & 1.6018 \\
\hline & Aug. & 11.98 & 1.4231 & 14.06 & 1.5589 & 12.23 & 1.4573 & 13.81 & 1.5609 \\
\hline & Mean & 22.61 & 1.9272 & 22.21 & 1.9521 & 22.63 & 1.9624 & 22.49 & 1.9885 \\
\hline \multirow{9}{*}{ Altay } & Jan. & 28.72 & 2.5901 & 25.89 & 2.3687 & 28.86 & 2.6122 & 25.27 & 2.3484 \\
\hline & Feb. & 14.60 & 1.3839 & 14.33 & 1.2246 & 15.99 & 1.5160 & 17.63 & 1.5045 \\
\hline & Mar. & 20.51 & 1.2551 & 25.87 & 1.5205 & 20.39 & 1.2970 & 24.29 & 1.5199 \\
\hline & Apr. & 23.46 & 1.2831 & 33.06 & 1.7122 & 24.50 & 1.3507 & 33.76 & 1.7828 \\
\hline & May. & 13.78 & 1.2706 & 14.66 & 1.3391 & 12.72 & 1.1755 & 12.54 & 1.1700 \\
\hline & Jun. & 7.14 & 0.8500 & 10.37 & 1.1788 & 7.99 & 0.9452 & 9.25 & 1.0982 \\
\hline & Jul. & 7.08 & 0.7932 & 7.00 & 0.7880 & 8.27 & 0.9380 & 8.36 & 0.9621 \\
\hline & Aug. & 7.66 & 0.7769 & 8.83 & 0.8922 & 9.33 & 0.9381 & 9.43 & 0.9508 \\
\hline & Mean & 15.37 & 1.2754 & 17.50 & 1.3780 & 16.01 & 1.3466 & 17.57 & 1.4171 \\
\hline
\end{tabular}


Table 4. Cont.

\begin{tabular}{|c|c|c|c|c|c|c|c|c|c|}
\hline \multirow{3}{*}{ Site } & \multirow{3}{*}{ Month } & \multicolumn{2}{|c|}{$A-F A C$} & \multicolumn{2}{|c|}{$A-S A C$} & \multicolumn{2}{|c|}{$M-F A C$} & \multicolumn{2}{|c|}{$M-S A C$} \\
\hline & & $M A P E$ & RMSE & MAPE & RMSE & $M A P E$ & RMSE & MAPE & RMSE \\
\hline & & $(\%)$ & $(\mathrm{m} / \mathrm{s})$ & $(\%)$ & $(\mathrm{m} / \mathrm{s})$ & $(\%)$ & $(\mathrm{m} / \mathrm{s})$ & $(\%)$ & $(\mathrm{m} / \mathrm{s})$ \\
\hline \multirow{9}{*}{ Korla } & Jan. & 33.06 & 2.8499 & 31.46 & 2.8438 & 33.21 & 2.8740 & 29.64 & 2.7286 \\
\hline & Feb. & 19.06 & 1.8813 & 16.62 & 1.5586 & 19.42 & 1.9041 & 19.24 & 1.7313 \\
\hline & Mar. & 43.74 & 1.7817 & 56.44 & 2.2029 & 45.87 & 1.9322 & 57.89 & 2.3821 \\
\hline & Apr. & 59.00 & 1.4235 & 44.51 & 1.2460 & 56.58 & 1.3678 & 43.84 & 1.1604 \\
\hline & May. & 33.43 & 3.2072 & 38.10 & 3.3685 & 36.05 & 3.3229 & 42.31 & 3.5991 \\
\hline & Jun. & 12.63 & 1.4397 & 12.99 & 1.5012 & 13.54 & 1.5625 & 15.78 & 1.8073 \\
\hline & Jul. & 14.50 & 1.8615 & 15.50 & 1.8892 & 16.29 & 2.0008 & 18.80 & 2.1764 \\
\hline & Aug. & 15.23 & 1.4691 & 16.23 & 1.5660 & 16.30 & 1.5598 & 17.46 & 1.6847 \\
\hline & Mean & 28.91 & 1.9892 & 28.98 & 2.0220 & 29.66 & 2.0655 & 30.62 & 2.1587 \\
\hline \multirow{9}{*}{ Hami } & Jan. & 51.74 & 2.5343 & 53.81 & 2.5421 & 51.74 & 2.5154 & 53.76 & 2.5358 \\
\hline & Feb. & 31.79 & 1.7927 & 32.06 & 1.6615 & 31.75 & 1.7633 & 34.41 & 1.7066 \\
\hline & Mar. & 52.34 & 1.3813 & 62.86 & 1.5416 & 54.02 & 1.4182 & 63.92 & 1.5989 \\
\hline & Apr. & 44.74 & 1.7330 & 47.42 & 1.7181 & 45.23 & 1.7231 & 46.41 & 1.7271 \\
\hline & May. & 17.44 & 2.2514 & 18.67 & 2.3539 & 17.80 & 2.2329 & 19.55 & 2.4045 \\
\hline & Jun. & 14.90 & 1.8122 & 16.21 & 1.9865 & 16.61 & 2.0506 & 19.64 & 2.4182 \\
\hline & Jul. & 11.14 & 1.4544 & 13.32 & 1.7891 & 12.80 & 1.7177 & 18.10 & 2.4109 \\
\hline & Aug. & 15.78 & 1.7640 & 16.29 & 1.7779 & 16.69 & 1.8476 & 16.97 & 1.8529 \\
\hline & Mean & 29.98 & 1.8404 & 32.58 & 1.9213 & 30.83 & 1.9086 & 34.09 & 2.0818 \\
\hline
\end{tabular}

To vividly illustrate the improved forecasting accuracy that is attributable to optimal parameter selection, we put the mean MAPE values of hybrid models integrated with and without the cuckoo search algorithm together in Table 5 for the four observation sites, namely Urumqi, Altay, Korla and Hami.

Table 5. The mean MAPE values (\%) of hybrid models with and without the CS algorithm.

\begin{tabular}{ccccc}
\hline Model & Urumqi & Altay & Korla & Hami \\
\hline$A-F A C$ & 22.61 & 15.37 & 28.91 & 29.98 \\
$A-F A C-C S$ & 12.94 & 13.77 & 18.08 & 21.64 \\
$A-S A C$ & 22.21 & 17.50 & 28.98 & 32.58 \\
$A-S A C-C S$ & 15.73 & 14.17 & 24.51 & 23.80 \\
$M-F A C$ & 22.63 & 16.01 & 29.66 & 30.83 \\
$M-F A C-C S$ & 12.88 & 14.92 & 18.99 & 22.17 \\
$M-S A C$ & 22.49 & 17.57 & 30.62 & 34.09 \\
$M-S A C-C S$ & 15.87 & 15.44 & 24.39 & 25.39 \\
\hline
\end{tabular}

As shown in Table 5, compared with hybrid forecasting strategies based on seasonal exponential adjustment techniques and adaptive coefficient methods with predetermined parameters, the further integrated cuckoo search algorithm contributes markedly to decreasing forecast errors. In particular, for Urumqi, the mean MAPE values of the eight months are reduced by $9.67 \%, 6.48 \%, 9.75 \%$ and $6.62 \%$ after the cuckoo search algorithm is introduced to the hybrid models, compared with A-FAC-CS, $A-S A C-C S, M-F A C-C S$ and $M-S A C-C S$, respectively. The comparisons of the forecast results regarding other three observation sites also indicate similar patterns, as shown in Table 5. Consequently, it can be concluded that the parameter optimization process should be regarded as a requisite component in the constructed hybrid forecast model. Moreover, the cuckoo search algorithm is a powerful search engine for parameters of the adaptive coefficient methods, and it is capable of improving forecast accuracy. 


\section{Conclusions}

This paper presents four novel kinds of hybrid models based on the seasonal exponential adjustment in the multiplicative or additive form, the first- or second-order adaptive coefficient methods and the cuckoo search algorithm for wind-speed forecasting. These models are significant because accurate wind-speed forecasting supplies essential information for wind-power assessment, wind-farm operations, integrated grid management and so on. To examine the effectiveness and superiority of the hybrid approaches proposed in this paper, daily mean wind-speed data sampled from four observation sites in Xinjiang Uygur Autonomous Region of China are taken into investigation. The simulation results described in Section 4 indicate that the developed innovative hybrid strategies provide satisfactory forecast accuracy.

As demonstrated in Section 4.1, there is sufficient evidence to show that the proposed hybrid models perform much better than existing individual forecasting approaches, such as the BP neural network, the ARIMA and the single adaptive coefficient method. Moreover, numerical experiments also suggest that introducing the $C S$ algorithm to the hybrid forecasting methods is highly recommended because it contributes remarkably to improving forecasting accuracy compared with the hybrid models using predetermined parameters.

We contribute to existing forecast model research by innovatively constructing hybrid forecasting models and integrating conventional adaptive coefficient models with an advanced cuckoo search algorithm. In particular, seasonal components and inherent trends are processed separately, which is regarded as an important data preprocessing technique in our research. In addition, the performance of the adaptive coefficient models is further improved by our work since we introduce the cuckoo search algorithm to conduct parameter optimization.

To sum up, considering their effectiveness and accuracy, the proposed hybrid forecasting strategies could be applied at large-scale wind farms to accomplish regular forecasting work. In addition, the proposed hybrid models for wind-speed forecasting also shed light on existing forecasting approaches for other variables, such as electricity demand and stock prices.

Author Contributions: Conceptualization, R.H. and Q.Y.; Methodology, R.H.; Software, Y.Y.; Validation, Q.Y., Y.C.; Formal Analysis, R.H. and Y.C.; Investigation, Y.C.; Resources, Y.Y.; Data Curation, Q.Y.; Writing-Original Draft Preparation, R.H.; Writing-Review \& Editing, R.H. and Y.Y.; Visualization, R.H. and Q.Y.; Supervision, Y.C.; Project Administration, Y.Y.; Funding Acquisition, Y.Y.

Funding: This research is supported by National Key R\&D Program of China (Grant number 2018YFB1003205, 2017YFE0111900) and 2019 Gansu key talent project.

Conflicts of Interest: The authors declare that there is no conflict of interest regarding the publication of this paper.

\section{Abbreviations}

$\begin{array}{ll}\text { ESN } & \text { Echo State Network } \\ \text { BP } & \text { Back Propagation } \\ \text { WNN } & \text { Wavelet Neural Network } \\ \text { GRNN } & \text { General Regression Neural Network } \\ \text { ANFIS } & \text { Adaptive Network-based Fuzzy Inference System } \\ \text { EEMD } & \text { Ensemble Empirical Mode Decomposition } \\ \text { SVM } & \text { Support Vector Machine } \\ \text { ELM } & \text { Extreme Learning Machine } \\ \text { WPD } & \text { Wavelet Packet Decomposition } \\ \text { ENN } & \text { Elman Neural Network } \\ \text { WPF } & \text { Wavelet Packet Filter } \\ \text { LSTM } & \text { Long Short Term Memory Network } \\ \text { CEEMDAN } & \text { Complete Ensemble Empirical Mode Decomposition with Adaptive Noise } \\ \text { SVR } & \text { Support Vector Regression } \\ \text { UKF } & \text { Unscented Kalman Filter }\end{array}$




\section{References}

1. Tomczewski, A.; Kasprzyk, L.; Nadolny, Z. Reduction of Power Production Costs in a Wind Power Plant-Flywheel Energy Storage System Arrangement. Energies 2019, 12, 1942. [CrossRef]

2. Zhang, W.; Wu, J.; Wang, J.; Zhao, W.; Shen, L. Performance analysis of four modified approaches for wind speed forecasting. Appl. Energy 2012, 99, 324-333. [CrossRef]

3. Zhao, Y.; Ye, L.; Li, Z.; Song, X.; Lang, Y.; Su, J. A novel bidirectional mechanism based on time series model for wind power forecasting. Appl. Energy 2016, 177, 793-803. [CrossRef]

4. Liu, H.; Mi, X.; Li, Y. Smart multi-step deep learning model for wind speed forecasting based on variational mode decomposition, singular spectrum analysis, LSTM network and ELM. Energy Convers. Manag. 2018, 159, 54-64. [CrossRef]

5. Qiu, X.; Zhang, L.; Suganthan, P.N.; Amaratunga, G.A. Oblique random forest ensemble via Least Square Estimation for time series forecasting. Inf. Sci. 2017, 420, 249-262. [CrossRef]

6. Ren, Y.; Suganthan, P.; Srikanth, N.; Amaratunga, G.; Suganthan, P. Random vector functional link network for short-term electricity load demand forecasting. Inf. Sci. 2016, 367, 1078-1093. [CrossRef]

7. Louka, P.; Galanis, G.; Siebert, N.; Kariniotakis, G.; Katsafados, P.; Pytharoulis, I.; Kallos, G. Improvements in wind speed forecasts for wind power prediction purposes using Kalman filtering. J. Wind Eng. Ind. Aerodyn. 2008, 96, 2348-2362. [CrossRef]

8. Wang, Y.; Hu, Q.; Meng, D.; Zhu, P. Deterministic and probabilistic wind power forecasting using a variational Bayesian-based adaptive robust multi-kernel regression model. Appl. Energy 2017, 208, 1097-1112. [CrossRef]

9. An, S.; Shi, H.; Hu, Q.; Li, X.; Dang, J. Fuzzy rough regression with application to wind speed prediction. Inf. Sci. 2014, 282, 388-400. [CrossRef]

10. Wang, S.; Zhang, N.; Wu, L.; Wang, Y. Wind speed forecasting based on the hybrid ensemble empirical mode decomposition and GA-BP neural network method. Renew. Energy 2016, 94, 629-636. [CrossRef]

11. Han, Q.; Wu, H.; Hu, T.; Chu, F. Short-Term Wind Speed Forecasting Based on Signal Decomposing Algorithm and Hybrid Linear/Nonlinear Models. Energies 2018, 11, 2976. [CrossRef]

12. Huang, Y.; Yang, L.; Liu, S.; Wang, G. Multi-Step Wind Speed Forecasting Based On Ensemble Empirical Mode Decomposition, Long Short Term Memory Network and Error Correction Strategy. Energies 2019, 12, 1822. [CrossRef]

13. Sun, S.; Wei, L.; Xu, J.; Jin, Z. A New Wind Speed Forecasting Modeling Strategy Using Two-Stage Decomposition, Feature Selection and DAWNN. Energies 2019, 12, 334. [CrossRef]

14. Blanchard, T.; Samanta, B. Wind Speed Forecasting Using Neural Networks. Wind Eng. 2019. Available online: https://doi.org/10.1177/0309524X19849846 (accessed on 23 September 2019).

15. Chitsazan, M.A.; Fadali, M.S.; Trzynadlowski, A.M. Wind speed and wind direction forecasting using echo state network with nonlinear functions. Renew. Energy 2019, 131, 879-889. [CrossRef]

16. Zhou, Q.; Wang, C.; Zhang, G. Hybrid forecasting system based on an optimal model selection strategy for different wind speed forecasting problems. Appl. Energy 2019, 250, 1559-1580. [CrossRef]

17. Hu, J.; Wang, J.; Zeng, G. A hybrid forecasting approach applied to wind speed time series. Renew. Energy 2013, 60, 185-194. [CrossRef]

18. Fu, W.; Wang, K.; Li, C.; Tan, J. Multi-step short-term wind speed forecasting approach based on multi-scale dominant ingredient chaotic analysis, improved hybrid GWO-SCA optimization and ELM. Energy Convers. Manag. 2019, 187, 356-377. [CrossRef]

19. Zhang, Z.; Qin, H.; Liu, Y.; Yao, L.; Yu, X.; Lu, J.; Jiang, Z.; Feng, Z. Wind speed forecasting based on Quantile Regression Minimal Gated Memory Network and Kernel Density Estimation. Energy Convers. Manag. 2019, 196, 1395-1409. [CrossRef]

20. Li, Y.; Shi, H.; Han, F.; Duan, Z.; Liu, H. Smart wind speed forecasting approach using various boosting algorithms, big multi-step forecasting strategy. Renew. Energy 2019, 135, 540-553. [CrossRef]

21. Wu, Y.-X.; Wu, Q.-B.; Zhu, J.-Q. Data-driven wind speed forecasting using deep feature extraction and LSTM. IET Renew. Power Gener. 2019, 13, 2062-2069. [CrossRef]

22. Cassola, F.; Burlando, M. Wind speed and wind energy forecast through Kalman filtering of Numerical Weather Prediction model output. Appl. Energy 2012, 99, 154-166. [CrossRef]

23. Niu, X.; Wang, J. A combined model based on data preprocessing strategy and multi-objective optimization algorithm for short-term wind speed forecasting. Appl. Energy 2019, 241, 519-539. [CrossRef] 
24. Chen, K.; Yu, J. Short-term wind speed prediction using an unscented Kalman filter based state-space support vector regression approach. Appl. Energy 2014, 113, 690-705. [CrossRef]

25. Guo, Z.; Wu, J.; Lu, H.; Wang, J.Z. A case study on a hybrid wind speed forecasting method using BP neural network. Knowl.-Based Syst. 2011, 24, 1048-1056. [CrossRef]

26. Zhu, S.; Wang, J.; Zhao, W.; Wang, J. A seasonal hybrid procedure for electricity demand forecasting in China. Appl. Energy 2011, 88, 3807-3815. [CrossRef]

27. Cadenas, E.; Jaramillo, O.A.; Rivera, W. Analysis and forecasting of wind velocity in chetumal, quintana roo, using the single exponential smoothing method. Renew. Energy 2010, 35, 925-930. [CrossRef]

28. De Faria, E.; Albuquerque, M.P.; González, J.; Cavalcante, J.; Albuquerque, M.P. Predicting the Brazilian stock market through neural networks and adaptive exponential smoothing methods. Expert Syst. Appl. 2009, 36, 12506-12509. [CrossRef]

29. Yang, X.S.; Deb, S. Cuckoo search via Lévy flights. In Proceedings of the World Congress on Nature and Biologically Inspired Computing (NaBIC), Coimbatore, India, 9-11 December 2009.

30. Yang, X.S. Nature-Inspired Metaheuristic Algorithms, 2nd ed.; Luniver Press: Frome, UK, 2010.

31. Yang, X.S.; Deb, S. Multiobjective cuckoo search for design optimization. Comput. Oper. Res. 2013, 40, 1616-1624. [CrossRef]

(C) 2019 by the authors. Licensee MDPI, Basel, Switzerland. This article is an open access article distributed under the terms and conditions of the Creative Commons Attribution (CC BY) license (http://creativecommons.org/licenses/by/4.0/). 